PBAR Note 668

\title{
What to Look For in the Accumulator Core Cooling Signal Suppression Measurements
}

\author{
Al Sondgeroth \\ Dave Vander Meulen \\ 01-15-02
}

This document explains what to look for in the signal suppression display produced by program P192 Pbar Accumulator Core Cooling Suppression Measurement. First we will give a quick overview of beam cooling and why we measure signal suppression.

Beam cooling is a technique whereby the physical size and energy spread of a particle beam circulating in a storage ring is reduced without any accompanying beam loss. The goal is to compress the same number of particles into a beam of smaller size and energy spread. This is desirable for the Accumulator as it compensates for various mechanisms leading to growth of beamsize and/or loss of stored particles and makes space available so that more beam can be stored. It also allows us to provide a low emittance beam through the Main Injector into the Tevatron in order to maximize the collision rate. For further information on specific stochastic cooling systems see Chapter 5 of the Antiproton Source Rookie Book.

Consider a single particle circulating in a storage ring. Assume that the particle has been injected with some error in position and angle with respect to the ideal orbit (the center of the beampipe). As the focusing system tries to restore the resultant deviation, the particle oscillates around the ideal orbit. These oscillations show up as betatron lines, or sidebands on a spectrum analyzer display. The cooling system is designed to damp the amplitude of these oscillations, or sidebands. A pickup array senses the position of the particle on each traversal. The signal is amplified through an amplifier chain and applied to the kicker array, which deflect the particle by an angle proportional to its error. The kickers and pickups along with the system gain, which includes the beam current, act as an op amp with negative feedback. An op amp with no feedback is shown in figure1.

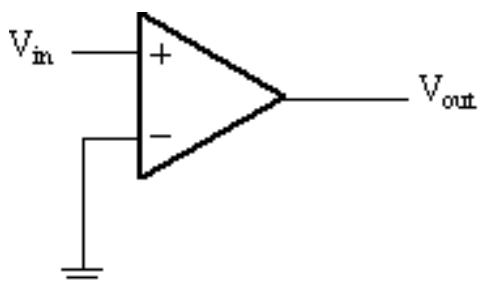

Figure 1 
PBAR Note 668

Figure 2 shows a typical op amp output response.

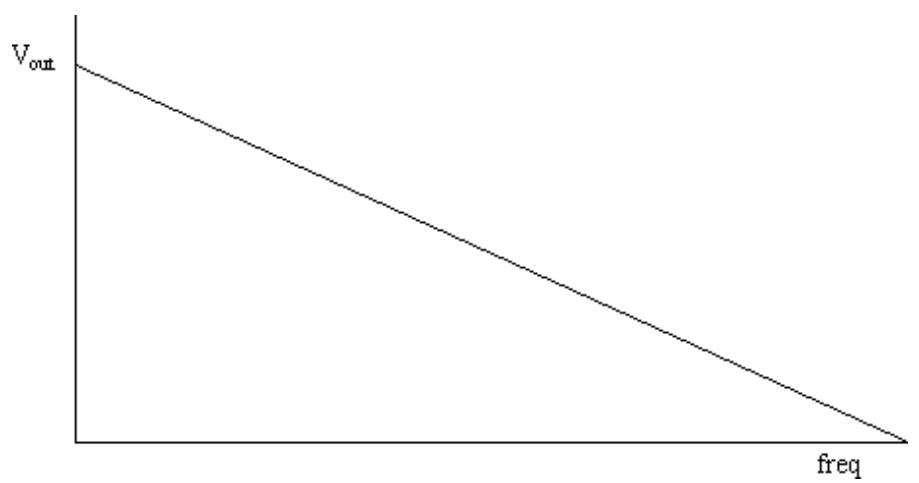

Figure 2

An op amp with negative feedback is shown in Figure 3.

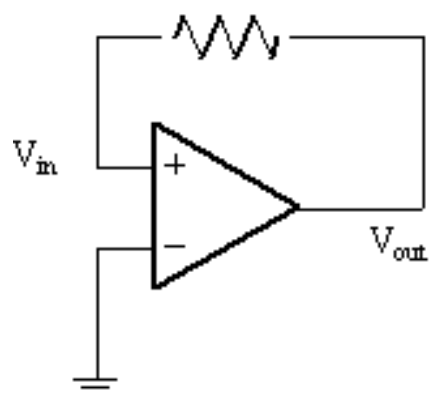

Figure 3

Figure 4 shows a typical op amp with negative feedback output response.

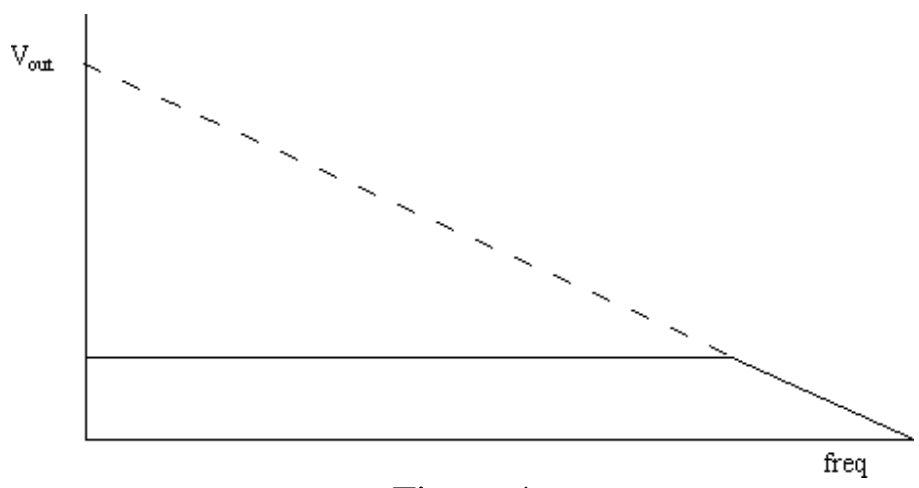

Figure 4

The feedback loop suppresses the signal. 
The system gain, which includes the beam, acts as the feedback portion of this circuit (Figure 5).

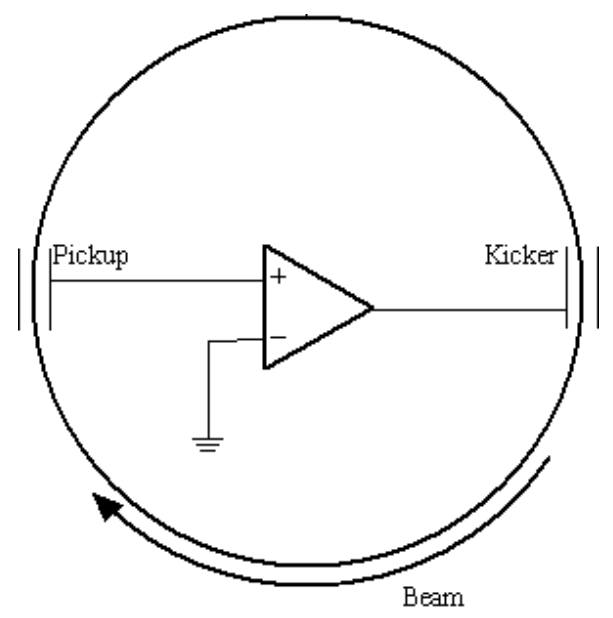

Figure 5

Refer to Figure 6. Note that the optimal cooling does NOT correspond to maximum power. The cooling process can be looked at as competition between two terms: (a) the coherent term, which is generated by a single particle, and, (b) the incoherent term, which results from disturbances to the single particle from its fellow sample particles through the feedback loop. The coherent signal's contribution to the cooling process is linearly proportional to the system gain while the incoherent heating term is proportional to the square of the system gain. The point where the two lines are the furthest apart is where the system will cool best and is known as the optimum gain. For our present core system, this point is roughly $4 \mathrm{~dB}$. Operationally, we like to run at this point.

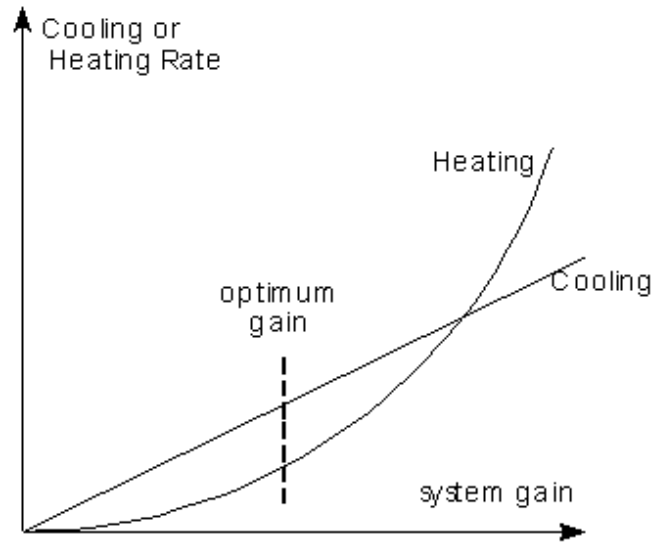

Figure 6 Variation of cooling and heating terms with system gain 
An attenuator is used to control the overall system gain and, hence, the amount of signal suppression. You will find that it is possible at times, especially with large stacks, to get more than the recommended $4 \mathrm{~dB}$ of signal suppression from the system. If we have a small stack the desired $4 \mathrm{~dB}$ of signal suppression may be hard or even impossible to achieve. Do not overdrive the cooling systems in an attempt to reach the optimal signal suppression. As the stack increases, the signal to noise of the system will improve and better signal suppression will be possible. The examples, used in this document, are shown with $142 \mathrm{~mA}$ of Pbars in the stack so $4 \mathrm{~dB}$ of signal suppression is easy to achieve.

The pickup arrays collect a signal from the beam. The collected signal has to arrive at the kicker array at the same time as the beam to achieve cooling. The path of the signal from the pickup array to the kicker array is shorter than the path of the beam and needs to be delayed. The signal is delayed using a trombone.

A PIN switch is used to turn the system on or off. See figure 7.

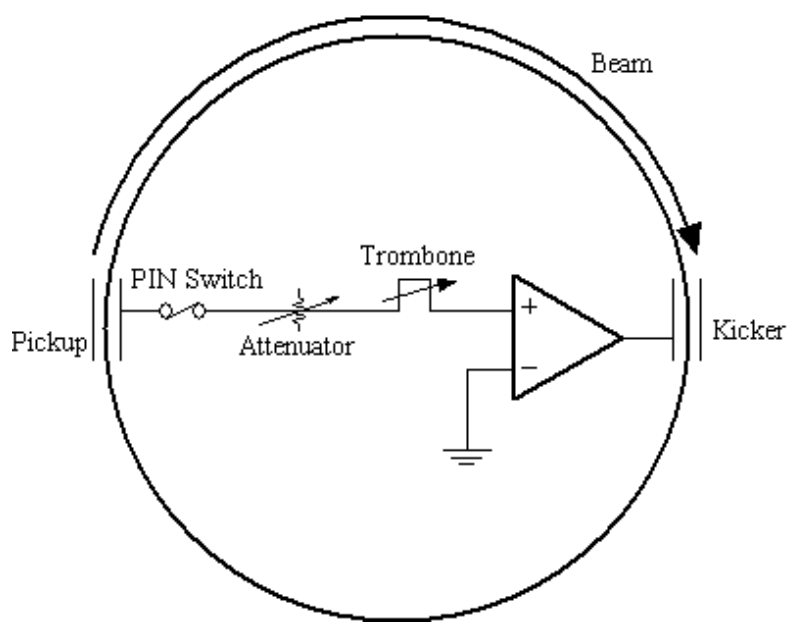

Figure 7

Table 1 lists specific cooling systems and their associated PIN switches, attenuators and trunk trombones.

System

2-4 Momentum

2-4 Horizontal

2-4 Vertical

4-8 Momentum

4-8 Horizontal

4-8 Vertical
PIN Switch

A:CPPS01

A:CHPS21

A:CVPS21

A:CMPS01

A:CHPS01

A:CVPS01
Attenuator

A:CPPA01

A:CHPA21

A:CVPA21

A:CMPA01

A:CHPA01

A:CVPA01
Trunk Trombone

A:СРTH01

A:CHTM21

A:CVTM21

A:CMTM01

A:CHTM01

A:CVTM01

Table 1 
As an example this document uses the 4-8 GHz core vertical transverse cooling system. Other core transverse system will be similar.

Figure 8 shows a display with sidebands, common mode and measurements labeled.

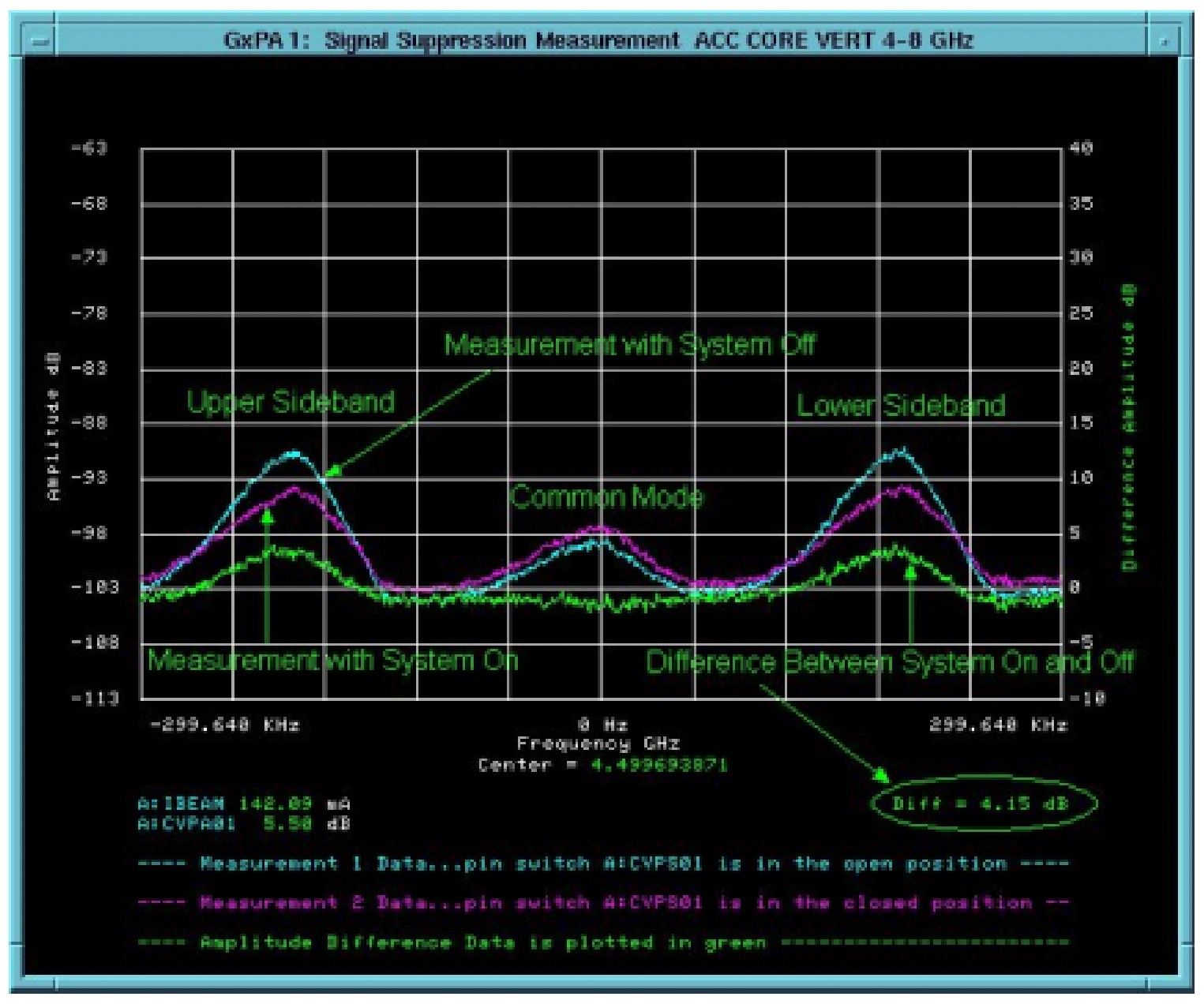

Figure 8

The common mode portion of this display is the longitudinal component of the schottky signal produced by the circulating beam. The common mode signals show up at each harmonic of the fundamental revolution frequency of the beam. The sidebands are the betatron oscillations around the ideal orbit and are used, among other things, to measure the horizontal and vertical betatron tunes of the machine. P192 measures only one band in the system. The system covers many bands. Note that the upper band is at a lower frequency than the common mode frequency displayed. The upper band displayed is actually the upper band for the next lower common mode harmonic. Similarly, the lower band displayed is the lower band for the next higher common mode harmonic. 
Figure 9 shows a spectrum analyzer measurement of a schottky signal over several harmonics $(\mathrm{H})$ with the common mode (CM), lower (LSB) and upper (USB) sidebands labeled and might help clarify the relationship between common mode harmonics and sidebands.

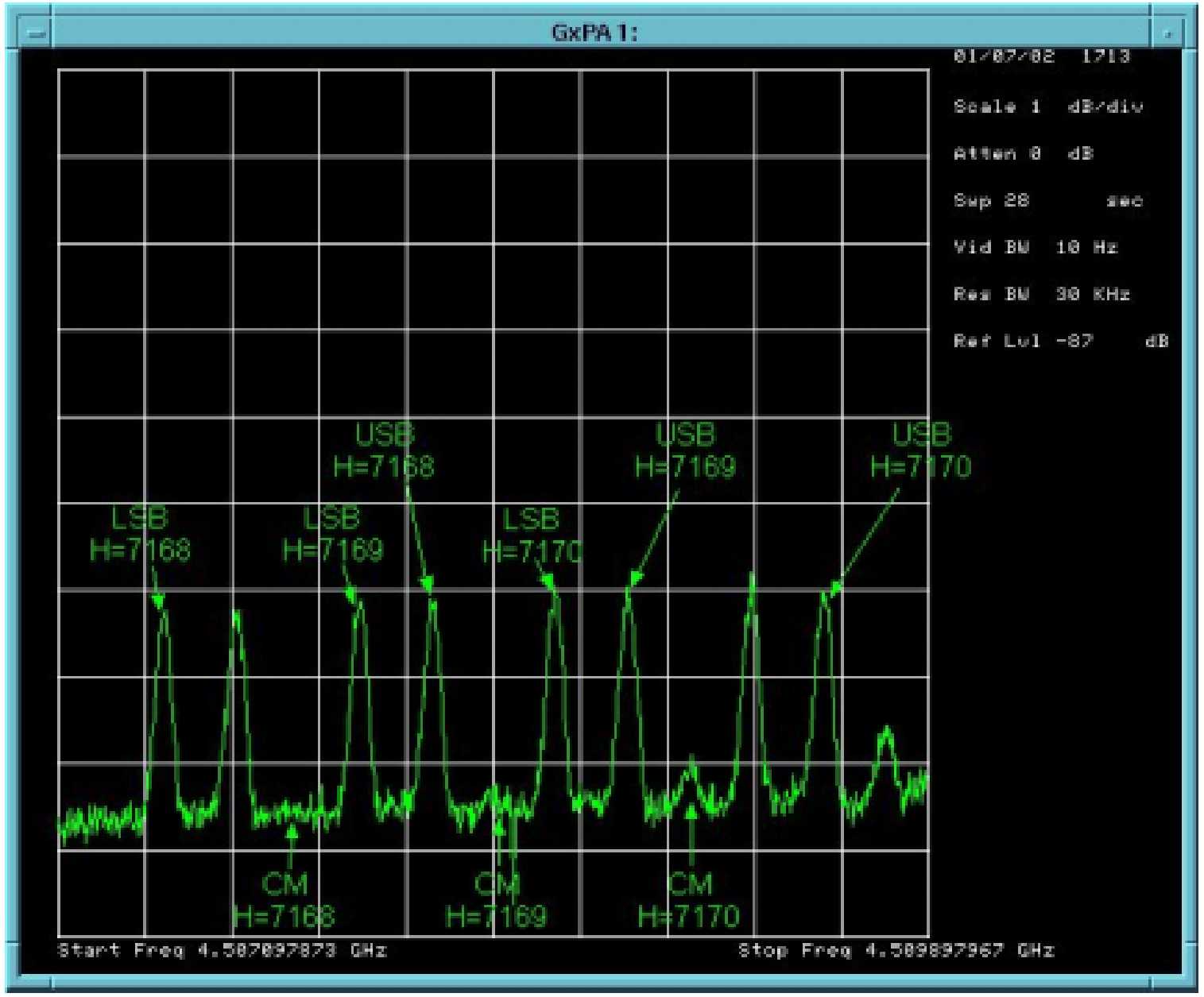

Figure 9 
Figure 10 shows the P192 display with the delay and amount of signal suppression are optimized.

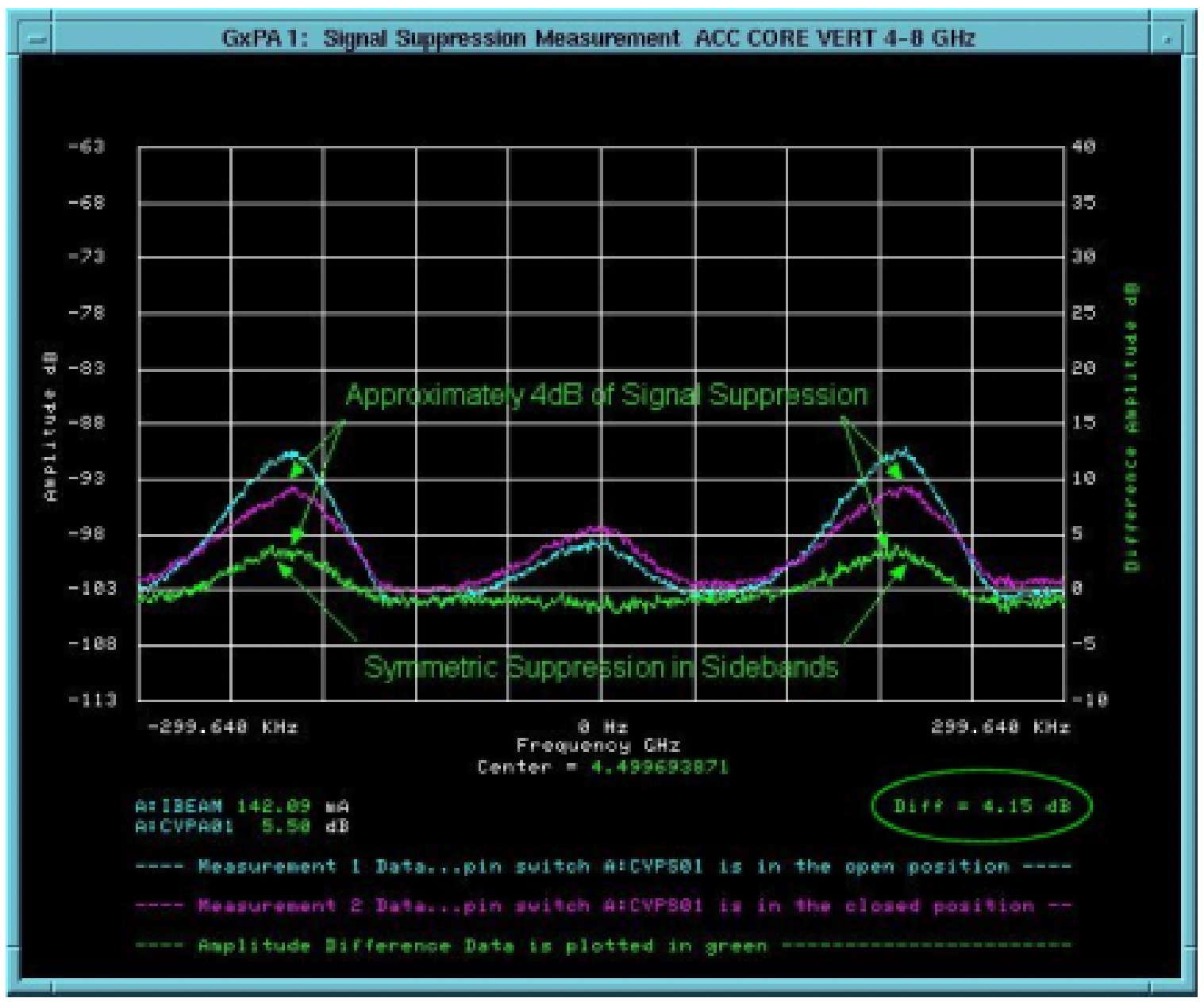

Figure 10 
If there is too much power in the cooling systems the P192 display will look like Figure 11 .

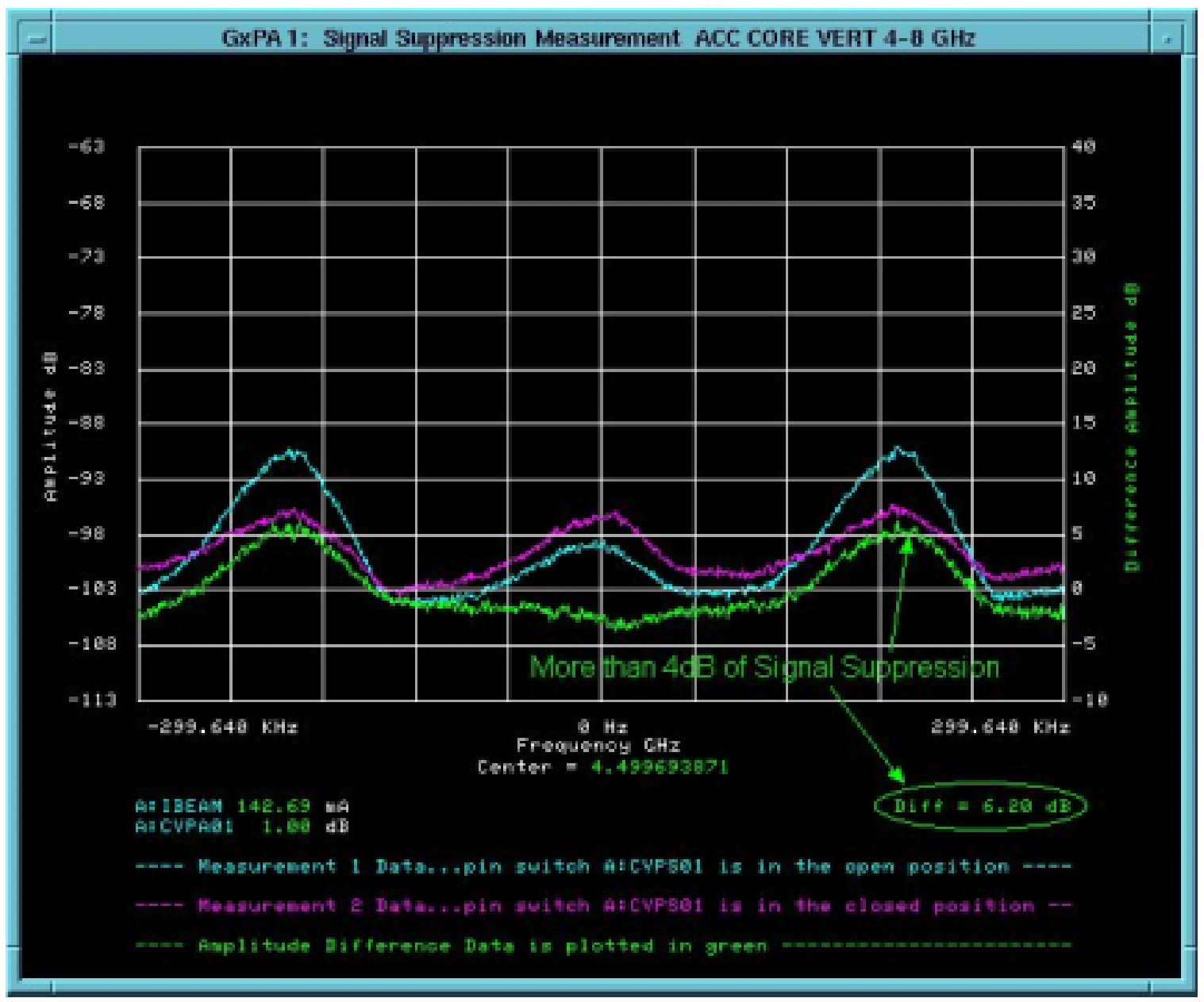

Figure 11

In the above scenario the attenuator, A:CVPA01 for our example, should be turned up until the signal suppression is back around $4 \mathrm{~dB}$. Every $3 \mathrm{~dB}$ increase in the attenuator will decrease the power in the system by $\sim 50 \%$. 
If the attenuator is set too high the display will look like Figure 12.

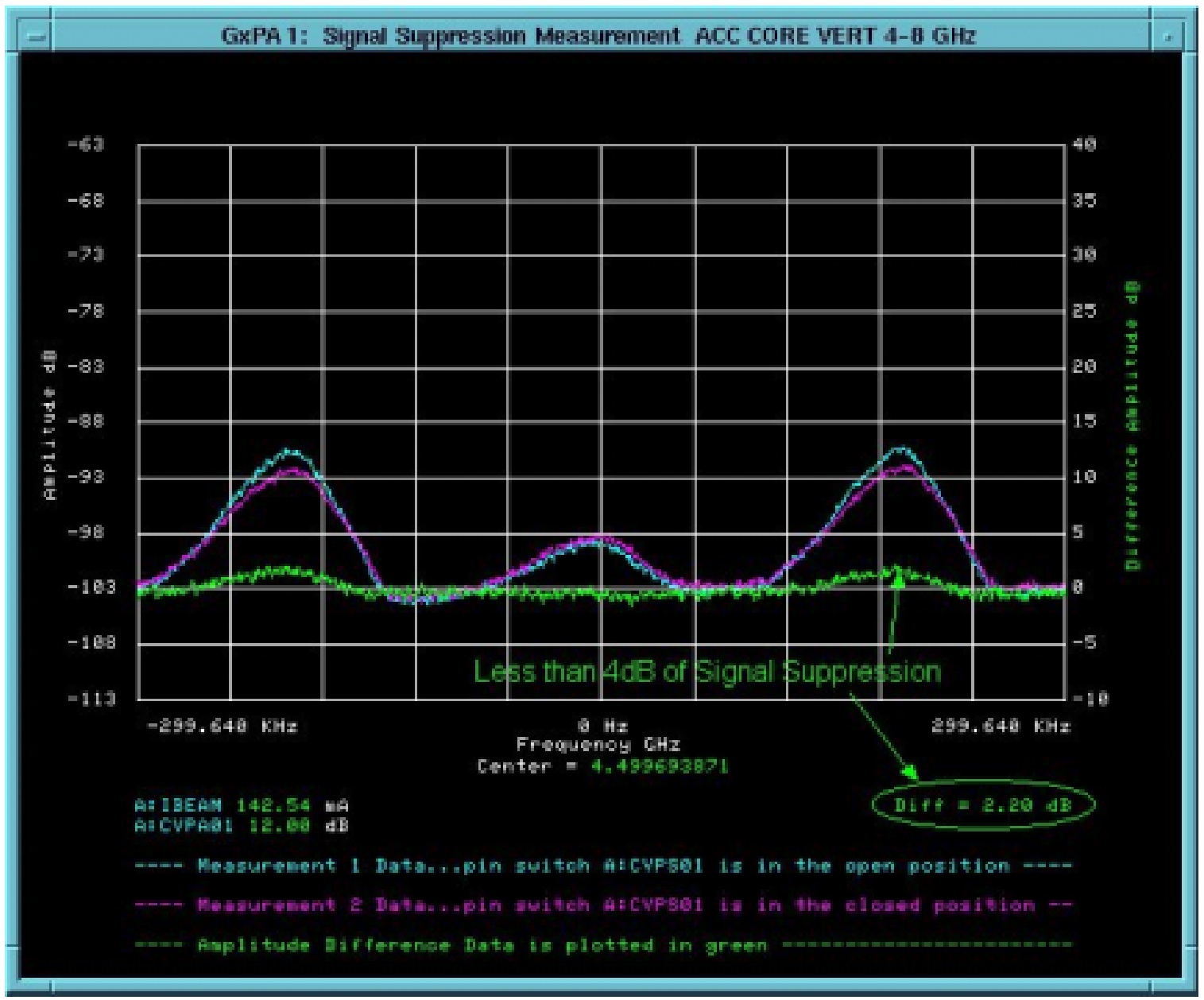

Figure 12

The attenuator should be lowered. Again, every $3 \mathrm{~dB}$ decrease in the attenuator will roughly double the power in the system. 
If the delay in the medium level trombone, in this case A:CVTM01, is too long the P192 display will look like Figure 12.

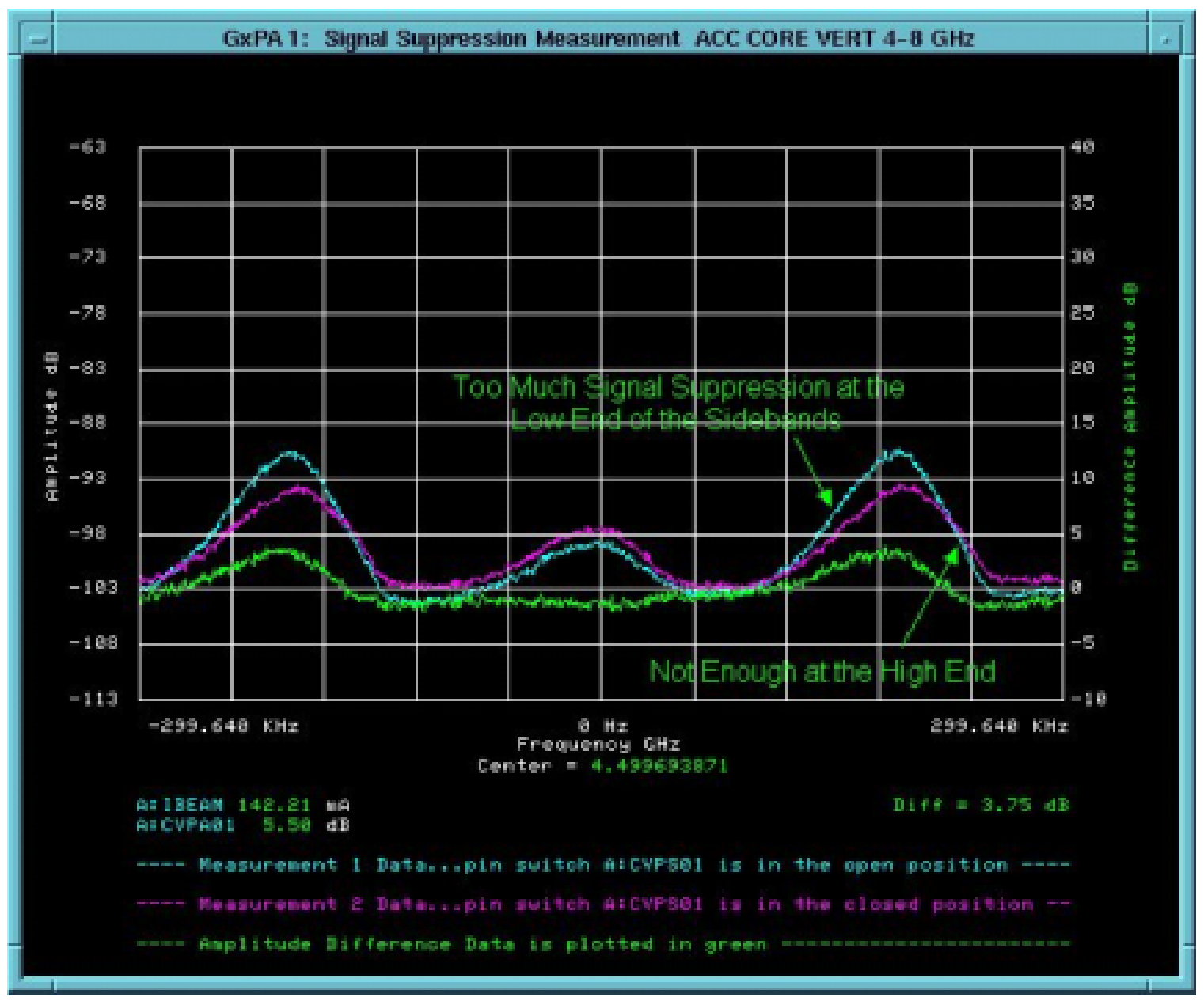

Figure 13

Some delay should be taken out of the corresponding medium level trombone. Generally, 10 ps changes are a good number to try. 
Figure 14 shows the P192 display with the delay too short.

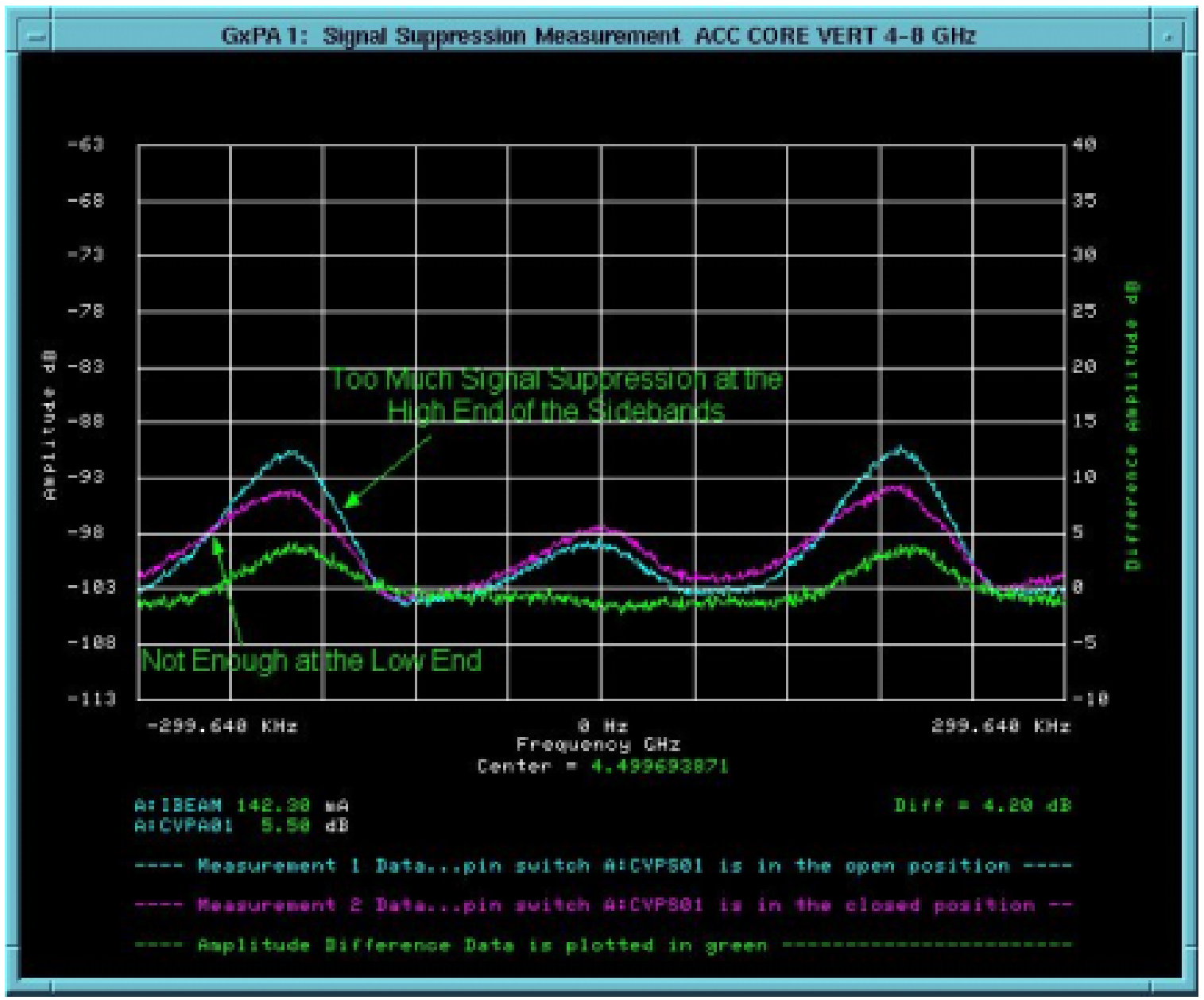

Figure 14

To correct this the delay in the trombone should be increased.

In summary, cooling is needed to store more beam and increase collision rates. Signal suppression is a measure of cooling efficiency. Attenuators are used to establish the optimum $4 \mathrm{~dB}$ of signal suppression when the accumulator stack is large enough and the medium level trombones are used to ensure the cooling system delay is in time with the beam. 